\title{
Pediatric obstructive sleep apnea syndrome - mini review
}

\begin{abstract}
Obstructive sleep apnea syndrome (OSAS) affects about $2 \%$ of children in the pre-school phase and can reach $12 \%$ in the pre-pubertal phase. The main clinical sign is snoring, and this should not be ignored. Characterized by nighttime respiratory arrest from total or upper airway obstruction, the disease leads to chronic sleep deprivation and consequently a series of metabolic, cognitive and behavioral changes in children. Treatment should occur as early and as effectively as possible, in an attempt to prevent the progression of the disease and its side effects in adult life. Adenotonsillectomy is gold standard treatment, providing an improvement in nasal and pharyngeal permeability. However, it does not correct morphological changes; it can lead to preoperative complications besides being costly. A more affordable, less invasive and less risky alternative treatment for children is needed. Studies addressing the rapid expansion of maxilla have been conducted; the results are promising but are still not well established in the literature.
\end{abstract}

Keywords: pediatric obstructive sleep apnea, primary snoring, adenoidectomy, tonsillectomy, polysomnography, upper airway resistance syndrome, rapid expansion of maxilla
Volume 8 Issue 4 - 2017

\author{
Thays Crosara Abrahao Cunha,' Thais De \\ Moura Guimaraes, ${ }^{2}$ Paulo Cesar Simamotto \\ Junior,' Thulio Marquez Cunha, ${ }^{3}$ Lia Rita \\ Azeredo Bittencourt ${ }^{2}$ \\ 'Department of Occlusion, Fixed Prosthesis and Dental \\ materials, Federal University of Uberlandia, Brazil \\ ${ }^{2}$ Department of Psychobiology, Federal University of Sao Paulo, \\ Brazil \\ ${ }^{3}$ Department of Pneumology, Federal university of Uberlandia, \\ Brazil
}

Correspondence: Thays Crosara Abrahao Cunha, Department of Occlusion, Fixed Prosthesis and Dental materials, Federal University of Uberl?ndia, Brazil, Rua Dos Pica Paus, 1750-Acaris 55-CEP: 38412-64I, Uberlandia, Brazil,

Email thayscrosara@gmail.com
Abbreviations: OSAS, obstructive sleep apnea syndrome; PSG, polysomnography; AHI, apnea and hipopnea index; CPAP, continuous positive airway pressure

\section{Introduction}

\section{Sleep}

Sleep is an activity that occupies one third to one half of our lives and is fundamental to good mental and emotional health, as well as being essential in the healthy maintenance of the body. Electroencephalographic patterns as well as the duration of different stages of sleep change throughout life. Children have different needs from adults, and adults, in turn, with different ages and stages of life, also have individual needs.

\section{Obstructive sleep apnea syndrome}

Obstructive Sleep Apnea Syndrome (OSAS) is classified as respiratory sleep disorder, affecting individuals of all ages, from neonates to the elderly. Recurrent episodes of total obstruction (apnea) or partial (hypopnea) of the upper airway simultaneously with respiratory effort during sleep lead to chronic sleep deprivation and the child suffers from all its consequences. ${ }^{1}$ The spectrum of the disease begins in snoring, progresses to resistance syndrome, obstructive sleep hypopnea until apnea due to total obstruction of the upper airway. ${ }^{2}$ Studies show that even children with only primary snoring already present behavioral changes and cognitive deficits when compared to healthy children. ${ }^{3,4}$ According to the International Classification of Sleep Disorders (CIDS-2), ${ }^{5}$ OSAS is mentioned in the category of "sleep-related respiratory disorders". This category is one of eight that describe such disorders. It classifies them according to their pathophysiological and clinical characteristics including the disorders of the adult, the child and the adolescent.

\section{Diagnostic method}

The International Classification of Sleep Disorders describes that the diagnosis of OSAS involves specific questionnaires, clinical history, physical examination and classical polysomnography (PSG). ${ }^{5}$ The use of PSG in a specialized laboratory with the accompaniment of trained professionals allows the evaluation of electroencephalogram (EEG), electro-oculogram (EOG), noninvasive electromyography (EMG) of the chin and lower limbs, oronasal flow measurements, thoracic-abdominal movement, electrocardiogram (ECG), pulse oximetry, and body position. With these records, we can calculate AHI (sleep apnea and hypopnea index per hour of sleep), oxyhemoglobin desaturation, percentages of sleeps stages; sleep efficiency and fragmentation, which can be attributed to respiratory events. It can be stated that PSG is the main objective method of diagnosis of OSAS. ${ }^{6}$ The severity of OSAS is based on its PSG indices; by the intensity of its symptoms; by its impact on social and professional functions, taking into account gender, age and profession; and by the presence of cardiovascular diseases. ${ }^{1}$ In children, OSAS is classified as mild when the patient presents AHI between 1 and 5 respiratory events per hour of sleep, moderate apnea for children presenting between 5 and 10 events/h and severe when recorded in PSG, more than 10 events per time of sleep. ${ }^{1}$

\section{Discussion}

The exact mechanism of this syndrome in children has not yet been fully elucidated, although important anatomical risk factors have been identified and are correlated with adjacent structures affecting the size and shape of the airway. Several techniques such as lateral neck radiography, cephalometry and magnetic resonance imaging have shown that the airway of children with apnea is lower than that of healthy children. ${ }^{78}$ During childhood, in the majority of cases, 
hypertrophy of the soft tissues attached to the upper airway, such as adenoid and palatine tonsils, may explain the clinical symptoms of children with OSAS and the surgical removal of these tissues may resolve or minimize this disorder. ${ }^{9}$ However, it is estimated that in 10 to $15 \%$ of children with apnea, this disorder cannot be treated by simply removing the palatine and adenoid tonsils. ${ }^{10}$ It is possible that the three-dimensional orientation of these tissues and how they overlap in the airway are preponderant factors and can significantly affect airway resistance. ${ }^{11}$ In 2001, Sanchez-Armengol and colleagues reported that the prevalence of OSAS was estimated at $1.9 \%$ for adolescents. ${ }^{12}$ It is not known if this disorder appears in adolescence as an extension of the alteration found in children, that is, where the main risk factor is adenotonsillar hypertrophy, or if it represents an early manifestation of the disease found in adults, being obesity and retrognathism mandibular risk factors. ${ }^{11}$ Some studies have discussed the relationship between sleep-disordered breathing and the developmental phase (childhood and adolescence). In a retrospective study, it was reported that this disorder was more frequent in adolescents who underwent adenotonsillectomy during childhood. ${ }^{13}$ On the other hand, Guilleminault et al. ${ }^{14}$ noticed changes in craniofacial morphology in adolescents with apnea and a history of upper airway obstruction during childhood. ${ }^{14}$ These authors also reported that the predisposition of these patients to this disorder would be related to genetic factors that would result in alterations in craniofacial growth or secondary alterations of the growth pattern in response to adenotonsillar hypertrophy. It is worth emphasizing the need for randomized studies to evaluate the long-term effects of treatments on the improvement of AHI levels as well as oxyhemoglobin saturation, considering the effect of infant growth, as well as the spontaneous resolution of the disease. ${ }^{15}$

\section{Consequences of OSAS}

\section{Clinical conditions}

Although adults with OSAS had a higher risk of cardiovascular morbidity and mortality, children with OSAS had elevated nocturnal blood pressure, diurnal systemic hypertension, changes in geometry and left ventricular function, as well as, endothelial changes. ${ }^{16}$ In addition, children with OSAS present maintained activation of the sympathetic nervous system, systemic inflammation and alteration in glucose and lipid metabolism, leading to the onset and spread of atherogenesis processes. ${ }^{16}$ The mechanisms that lead to these changes appear to be related to the interaction of intermittent hypoxia, hypercapnia, frequent awakenings and variations in intrathoracic pressures.

\section{Quality of life}

Quality of life is one of the parameters that has been used to evaluate the impact of a disease or a particular comorbidity in the life of the individual, as well as the effect of treatment, not only according to oxidative markers, but also according to perception of the individual. Garetz SL. ${ }^{17}$ have tracked literature studies that analyzed aspects of behavior, cognition and quality of life in children with OSAS before and after adenotonsillectomy. ${ }^{17} 25$ articles were screened, of these, despite the different diagnostic methodologies and a large variability sample (from 19 to 297 subjects), all studies showed an increase in one or more of the measures evaluated, that is, changes in quality of life; improvement in behavior, especially hyperactivity indexes and aggression problems; improvement of scores on tasks of memory, attention and academic performance. ${ }^{17}$

\section{Treatments for pediatric OSAS}

Immediate treatment of the onset of OSAS symptoms can prevent adverse effects on vital functions, thus demonstrating the need for simpler, easier access and low cost diagnostic exams. ${ }^{18}$

\section{Adenotonsillectomy}

Differently from adults with OSAS, where CPAP (Continuous Positive Airway Pressure) is the most effective treatment, adenotonsillectomy is the main treatment in children with the disease. Intervention studies demonstrated, after adenotonsillectomy, short-term improvement of respiratory variables in PSG in $83 \%$ of patients. ${ }^{19}$ In addition, there is improvement of lipid metabolism and inflammatory variables. ${ }^{20}$ Although this method is the most effective treatment for OSA in children, surgery does not improve morphological changes in the oral breathing infant and may lead to preoperative complications. ${ }^{21}$ Children with OSA who undergo general anesthesia and adenotonsillectomy frequently present postoperative respiratory discomfort and even death. Therefore, they require preoperative monitoring in an intensive care unit. ${ }^{18}$ Although this surgery improves nasal and pharyngeal permeability, it does not correct jaw hypotonic, elevated hard palate, postural and masticatory changes. This surgery in children, resolves between 40 to $60 \%$ of cases. Therefore, the needs for other treatments that serve as a primary or complementary therapeutic option are necessary. ${ }^{22}$ We also emphasize the importance of identifying a more accessible alternative, less invasive and less risky for children; one option has been the rapid expansion of the maxilla.

\section{Rapid Maxillary Expansion}

Rapid maxillary expansion promotes an osteogenic distraction in the upper bone base. This distraction at the level of the palatine suture expands the maxilla, increasing its transverse dimension, as well as the volume space of the nasal cavity, providing an improvement in the respiratory distress index in children. ${ }^{23,24}$ The maxillary disjunction when associated with mandibular advancement directly promotes an increase in airspace volume by improving airflow limitation events in adolescents. ${ }^{25}$ On the other hand, indirectly, the disjunction of the maxilla, due to the increase in the volume of the buccal and nasal cavity, associated with the anterior repositioning of the mandible, results in an increase in the or pharyngeal space by modifying the resting position of the tongue promoting the return of normal masticatory function. ${ }^{26}$ In isolation, the rapid maxillary expansion was evaluated by Villa et al..$^{27}$ in the treatment of children with signs and symptoms of OSAS. After 12 months of follow-up, $92.8 \%$ of these patients ceased to be mouth breathers, which according to these authors suggest that the nasal cavity was increased. Other authors also found a reduction in nasal resistance after treatment of children and adolescents with rapid maxillary expansion. ${ }^{28}$ Reducing nasal resistance is one of the main goals of rapid maxillary expansion and was documented by Timms DJ. ${ }^{29}$ through rhinonanometry. In order to evaluate the instability of non-REM sleep before and after MRS, Villa et al. ${ }^{27}$ evaluated 9 children between 4 and 8years of age with sleep apnea. ${ }^{28}$ Although maxillary expansion has provided a normalization of sleep architecture and improved respiratory disorders at night, the sleep microstructure and respiratory parameters have not been fully reestablished, demonstrating that this is a suboptimal treatment for the disease. ${ }^{28}$ However, to date, we do not know of other studies that have evaluated the microstructure of sleep before and after not only ERM but also adenotonsillectomy or CPAP. This demonstrates the 
lack of studies also addressing this parameter. Villa MP. ${ }^{31}$ observed that rapid maxillary expansion can be used to treat apnea in children with malocclusion and its effects persist until 24 months after the end of the intervention. ${ }^{31}$ Barreto and collaborators (2014) selected 52 children with OSAS and divided them into 3 groups. ${ }^{32}$ Those with a milder disease were referred for MRA and those with a more severe disease were referred for amygdala and adenoid surgery. After the interventions, the reduction of AHI was observed in both procedures, but the children who underwent surgery and who still had apnea were submitted to MRA, establishing the third treatment group. There was an improvement in the clinical picture of OSAS in the three groups treated. According to the authors, the onset age and severity of OSAS should be the main factors involved in the choice of treatment.

\section{Conclusion}

Although not well established in the literature, both the rapid expansion of maxilla and adenotonsillectomy has the potential to improve the clinical picture of the child with OSAS. However, more studies are necessary for MRA to be adopted as an alternative exclusive for the treatment of OSAS. Studies that evaluate the craniofacial characteristics of the possible responders, as well as their long-term effect.

\section{Acknowledgments}

FOUFU, FAPEMIG, CAPES, CNPq.

\section{Conflicts of interest}

The authors don't have conflict of interest to disclosures.

\section{Funding}

None.

\section{References}

1. Sleep-related breathing disorders in adults: recommendations for syndrome definition and measurement techniques in clinical research. The report of an American Academy of Sleep Medicine Task Force. Sleep. 1999;22(5):667-689.

2. Sargi Z, Younis R. Pediatric Obstruvtive Sleep Apnea: Current Management. ORL J Otorhinolaryngol Relat Spec. 2007;69(6):340-344.

3. O'Brien LM, Mervis CB, Holbrook CR, et al. Neurobehavioral implications of habitual snoring in children. Pediatrics. 2004;114(1):44-49.

4. Blunden S, Lushington K, Kennedy D, et al. Behavior and neurocognitive performance in children aged 5-10 years who snore compared to controls. J Clin Exp Neuropsychol. 2000;22(5):554-568.

5. American Academy of Sleep Medicine. The International Classification of Sleep Disorders: diagnostic and coding manual. $2^{\text {nd }}$ edn. American Academy of Sleep Medicine, USA; 2005. 1-297 p.

6. Vale F, Albergaria M, Carrilho E, et al. Efficacy of Rapid Maxillary Expansion in the Treatment of Obstructive Sleep Apnea Syndrome: A Systematic Review With Meta-analysis. $J$ Evid Based Dent Pract. 2017;17(3):159-168.

7. Arens R, McDonough JM, Costarino AT, et al. Magnetic resonance imaging of upper airway structure of children with obstructive sleep apnea syndrome. Am J Respir Crit Care Med. 2001;164(4):698-703.

8. Gozal D, Burnside MM. Increased upper airway collapsibility in children with obstructive sleep apnea during wakefulness. Am J Respir Crit Care Med. 2004;169(2):163-167.
9. John S Suen, James E Arnold, Lee J Brooks. Adenotonsillectomy for Treatment of Obstructive Sleep Apnea in Children. Arch Otolaryngol Head Neck Surg. 1995;121(5):525-530.

10. Tal A, Bar A, Leiberman A, et al. Sleep characteristics following adenotonsillectomy in children with obstructive sleep apnea syndrome. Chest. 2003;124(3):948-953.

11. Arens R, Marcus CL. Pathophysiology of upper airway obstruction: a developmental perspective. Sleep. 2004;27(5):997-1019.

12. Sánchez-Armengol A, Fuentes-Pradera MA, Capote-Gil F, et al. Sleeprelated breathing disorders in adolescents aged 12 to 16 years: clinical and polygraphic findings. Chest. 2001;119(5):1393-1400.

13. Morton S, Rosen C, Larkin E, et al. Predictors of sleep-disordered breathing in children with a history of tonsillectomy and/or adenoidectomy. Sleep. 2001;24(7):823-829.

14. Guilleminault C, Rafael Pelayo, Damien Leger, et al. Recognition of sleep-disordered breathing in children. Pediatrics. 1996;98(5):871- 882.

15. Camacho M, Chang ET, Song SA, et al. Rapid maxillary expansion for pediatric obstructive sleep apnea: A systematic review and meta-analysis. Laryngoscope. 2017;127(7):1712-1719.

16. Capdevilla OS, Kheirandish-Gozal L, Dayyat, et al. Pediatic obstructive sleep apnea. Complications, management and long term outcomes. Proc Am Thorac Soc. 2008;5(2):274-282.

17. Garetz SL. Behavior, cognition, and quality of life after adenotonsillectomy for pediatric sleep-disordered breathing: Summary of the literature. Otolaryngology Head and Neck Sur. 2008;138(1 Suppl):S19-S26.

18. American Academy of Pediatrics (AAP). Clinical practice guideline: Diagnosis and management of childhood obstructive sleep apnea syndrome. Section on Pediatric Pulmonology. Subcommittee on Obstructive Sleep Apnea Syndrome. Pediatrics. 2002;109(4):704-712.

19. Brietzke SE, Gallagher D. The effectiveness of tonsillectomy and adenoidectomy in the treatment of pediatric obstructive sleep apnea/ hypopnea syndrome: a meta-analysis. Otolaryngol Head Neck Surg. 2006;134(6):979-984.

20. Gozal D, Capdevila OS, Kheirandish-Gozal L. Metabolic alterations and systemic inflammation in obstructive sleep apnea among nonobese and obese prepubertal children. Am J Respir Crit Care Med. 2008;177(10):1142-1149.

21. McColley SA, April MM, Carroll JL, et al. Respiratory compromise after adenotonsillectomy in children with obstructive sleep apnea. Arch Otolaryngol Head Neck Surg. 1992;118(9):940-943.

22. Mylavarapu G, Subramaniam D, Jonnagiri R, et al. Computational Modeling of Airway Obstruction in Sleep Apnea in Down Syndrome: A Feasibility Study. Otolaryngol Head Neck Surg. 2016;155(1):184-187.

23. Pirelli P, Saponara M, Guilleminault C. Rapid maxillary expansion in children with obstructive sleep apnea syndrome. Sleep. 2004;27(4):761-766.

24. Principato JJ. Upper airway obstruction and craniofacial morphology. Otolaryngol Head Neck Surg. 1991;104(6):881-890.

25. Schütz TC, Dominguez GC, Hallinan MP, et al. Class II correction improves nocturnal breathing in adolescents. Angle Orthod. 2011;81(2):222-228.

26. Pirelli P, Marullo M, Casagrande M. Espansione rapida del mascellare: effetti sulla funzionalità respiratória ed uditiva. Mondo Ortod. 1995;20:129-135.

27. Villa MP, Malagola C, Pagani J, et al. A Rapid maxillary expansion in children with obstructive sleep apnea syndrome: 12 month follow-up. Sleep Med. 2007;8(2):128-134. 
28. Bicakci AA, Agar U, Sökücü O, et al. Nasal airway changes due to rapid maxillary expansion timing. Angle Orthod. 2005;75(1):1-6.

29. Timms DJ. Rapid maxillary expansion in the treatment of nocturnal enuresis. Angle Orthod. 1990;60(3):229-234.

30. Villa MP, Bernkopf E, Pagani J, et al. Randomized controlled study of an oral jaw-positioning appliance for the treatment of obstructive sleep apnea in children with malocclusion. Am J Respir Crit Care Med. 2002;165:123-127.
31. Villa MP, Rizzoli A, Miano S, et al. Efficacy of rapid maxillary expansion in children with obstructive sleep apnea syndrome: 36 months of followup. Sleep Breath. 2011;15(2):179-184.

32. Villa MP, Castaldo R, Miano S, et al. Adenotonsilectomy and orthodontic therapy in pediatric obstructive sleep apnea. Sleep Breath. 2014;18(3):533-539. 